\title{
INCONTINENCE
}

\section{Intravesical 'shock absorber' provides relief from SUI}

Innovative researchers in the USA have created a device that acts as a 'shock absorber' in the bladder to reduce the symptoms of stress urinary incontinence (SUI). They hope their device will provide an effective, minimally invasive alternative to current treatment options, and the results of their prospective, multicentre, randomized controlled trial of the device-recently published in The Journal of Urology - seem to support this idea.

\section{4 ...a gas-filled balloon that sits in the bladder and absorbs spikes of pressure... 77}

Inspired by industrial hydraulic systems, the research team came up with the concept of a gas-filled balloon that sits in the bladder and absorbs spikes of pressure that are transmitted from the abdomen during physical activity. Their prototype is made of biocompatible medical grade polyurethane and is inserted via the urethra without anaesthesia or analgesia. Once placed in the bladder, the balloon is inflated with $15 \mathrm{~cm}^{3}$ of air and $0.5 \mathrm{~cm}^{3}$ of a perfluorocarbon liquid that prevents diffusion of air through the polyurethane. A one-way valve seals the balloon after inflation. The device has a low mass $(0.2 \mathrm{~g})$ and inherent buoyancy, enabling it to float at the dome of the bladder rather than occluding the bladder outlet during voiding.

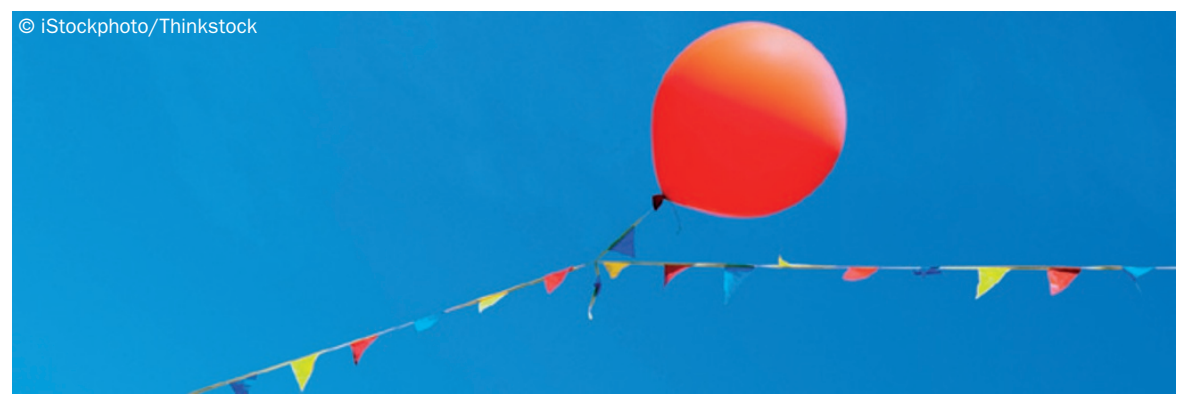

166 women with SUI symptoms (demonstrated on urodynamics or by cough test) were recruited at 13 sites across the USA and randomized to receive either the pressure-attenuation device $(n=112)$ or sham procedure $(n=54)$. The device was changed (or sham procedure repeated) every 90 days. After 6 months, women who received the device had significantly improved symptoms compared with those in the control arm, based on the number of women who reached a composite end point of $\geq 50 \%$ reduction in provocative pad weight test and a subjective measure of improvement in urine leakage (50.8\% versus $16.3 \% ; P<0.001)$. This improvement was also evident when the data were analysed on an intent-to-treat basis (32\% versus $15 \% ; P<0.001$ ).

Sediment formation can be a major concern with intravesical devices, so the investigators checked 539 devices visually for sediment formation and measured the thickest deposit at its thickest point. 89.4\% of devices had no measurable sediment formation $(>0.1 \mathrm{~mm})$, and there were no reported cases of obstruction or urinary retention. Complication rate was quite high with a total of 227 events reported in 99 patients in the treatment group (88.4\%), compared with 22 in the control group (40.7\%). However, only five serious adverse events were reported overall.

Unfortunately, the withdrawal rate was much higher than the researchers had expected, with 51 patients leaving the trial in the first 6 months (30.7\%), most commonly owing to issues with the device or delivery system. The investigators are now analysing their data to identify factors that might predict tolerability and improve patient selection. Perhaps after some tweaks to the design and insertion procedure, this pressure-attenuation device will be a promising option for the treatment of SUI in the future.

Sarah Payton

Original article Rovner, E. S. et al. A randomized, controlled clinical trial of a novel intravesical pressure-attenuation device for the treatment of stress urinary incontinence. J. Urol. doi:10.1016/j.juro.2013.06.042 